\title{
Epigenetic-sensitive liquid biomarkers and personalised therapy in advanced heart failure: a focus on cell-free DNA and microRNAs
}

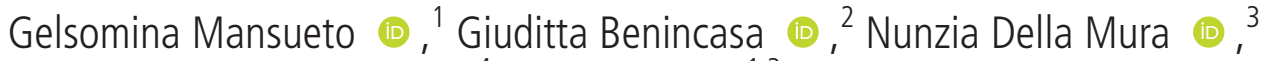 \\ Giovanni Francesco Nicoletti, ${ }^{4}$ Claudio Napoli ${ }^{1,3}$
}

\begin{abstract}
${ }^{1}$ Clinical Department of Internal Medicine and Specialistics, Department of Advanced Medical and Surgical Sciences (DAMSS), University of Campania "Luigi Vanvitelli", Naples, Italy ${ }^{2}$ Department of Advanced Medical and Surgical Sciences (DAMSS), University of Campania "Luigi Vanvitelli", Naples, Italy

${ }^{3}$ IRCCS SDN, Naples, Italy ${ }^{4}$ Multidisciplinary Department of Medical, Surgical and Dental Sciences, Plastic Surgery Unit, University of Campania "Luigi Vanvitelli", Naples, Italy
\end{abstract}

\section{Correspondence to}

Dr Giuditta Benincasa, Clinical Department of Internal Medicine and Specialistics, Department of Advanced Clinical and Surgical Sciences, University of Campania Luigi Vanvitelli, Naples 80132, Italy; dr. benincasa.giuditta@gmail.com

Received 19 December 2019 Revised 7 March 2020

Accepted 4 April 2020 Published Online First 2 July 2020

\section{ABSTRACT}

Dilated cardiomyopathy (DCM) represents a common genetic cause of mechanical and/or electrical dysfunction leading to heart failure (HF) onset for which truncating variants in titin (TTN) gene result in the most frequent mutations. Moreover, myocyte and endothelial cell apoptosis is a key endophenotype underlying cardiac remodelling. Therefore, a deeper knowledge about molecular networks leading to acute injury and apoptosis may reveal novel circulating biomarkers useful to better discriminate HF phenotypes, patients at risk of heart transplant as well as graft reject in order to improve personalised therapy. Remarkably, increased plasma levels of cell-free DNA (cfDNA) may reflect the extent of cellular damage, whereas circulating mitochondrial DNA (mtDNA) may be a promising biomarker of poor prognosis in patients with HF. Furthermore, some panels of circulating miRNAs may improve the stratification of natural history of disease. For example, a combination of miR-558, miR-122* and miR-520d-5p, as well as miR125a-5p, miR-550a-5p, miR-638 and miR-190a, may aid to discriminate different phenotypes of HF ranging from preserved to reduced ejection fraction. We give update on the most relevant genetic determinants involved in DCM and discuss the putative role of non-invasive biomarkers to overcome current limitations of the reductionist approach in HF management.

\section{INTRODUCTION}

Heart failure (HF) is considered a pandemic disease with a high number of symptomatic adults, high mortality and expansive cost, representing the main cause of hospitalisation of patients over 65 years, with or without comorbidities. ${ }^{1-3} \mathrm{HF}$ is characterised by structural and functional injury leading to reduced cardiac output and/or elevated intracardiac pressure at rest or during stress which are associated with common symptoms, including breathlessness, ankle swelling and fatigue, and signs, including elevated jugular venous pressure, pulmonary crackles and peripheral oedema. ${ }^{4}$ According to the latest guidelines published from the American College of Cardiology/American Heart Association, ${ }^{1} \mathrm{HF}$ comprises three subgroups of patients, including those with normal left ventricular (LV) ejection fraction $(\geq 50 \%$; preserved ejection fraction, $\mathrm{HFpEF}$ ), those with reduced LV ejection fraction ( $<40 \%$; reduced ejection fraction, HFrEF), as well as patients with mid-range ejection fraction (40\%-49\%, HFmrEF). ${ }^{1}$ Generally, diagnosis of
HFpEF is more challenging because patients have normal LV chamber size and greater wall thickness and/or increased left atrial (LA) size, as a sign of increased filling pressure. ${ }^{1}$ Cardiomyopathies lead to different clinical symptoms ranging from $\mathrm{HF}$, life-threatening arrhythmias and sudden cardiac death (SCD). Moreover, many cellular, molecular and humoral mechanisms are activated to restore pressure and redistribution of the circle in both preload and postload. ${ }^{15}$ A clear distinction between acquired and inherited cardiomyopathies remains challenging. ${ }^{6}$ Despite genetic counselling is recommended in patients with hypertrophic cardiomyopathy (HCM), arrhythmogenic right ventricular cardiomyopathy and idiopathic dilated cardiomyopathy (DCM), there is no confirmatory role for routine genetic testing in the most of patients with a definite clinical diagnosis of HF. Cellular mechanisms and molecular networks causing the progressive deterioration of myocardial function in $\mathrm{HF}$ remain not fully clarified, also. However, it may result from both programmed cell death (apoptosis) and necrosis releasing different circulating biomarkers and inflammatory mediators (eg, cytokines), with established clinical role in laboratory detection of HF onset and prediction of disease progression. ${ }^{5}$ Thus, a deeper knowledge of complex apoptosis-related regulatory pathways is needed to establish novel non-invasive biomarkers to identify early lesions and monitor the risk of allograft rejection. Since apoptosis is a highly regulated process with respect to necrosis, it also represents a putative therapeutic target when intervention occurs at early stages of disease. ${ }^{5}$

Epigenetic-sensitive mechanisms play a key role in regulating apoptosis and necrosis in the heart. ${ }^{57}$ The major epigenetic mechanisms are DNA methylation, histone and non-histone modifications, and non-codingRNA molecules, mainly microRNA (miRNAs) which control gene expression without altering DNA sequence in several cardiovascular diseases. ${ }^{8-13}$ Remarkably, advanced next-generation sequencing (NGS) platforms emphasised the putative useful role of liquid-based assays for detecting cell-free DNA (cfDNA) and miRNAs to improve precision medicine of HF. ${ }^{11} 14 \quad 15$ We briefly summarise genetic determinants involved in DCM diagnosis and progression of HF (figure 1). Moreover, we focus on the putative role of circulating cfDNA and miRNAs derived from cardiomyocyte damage as promising non-invasive biomarkers of 


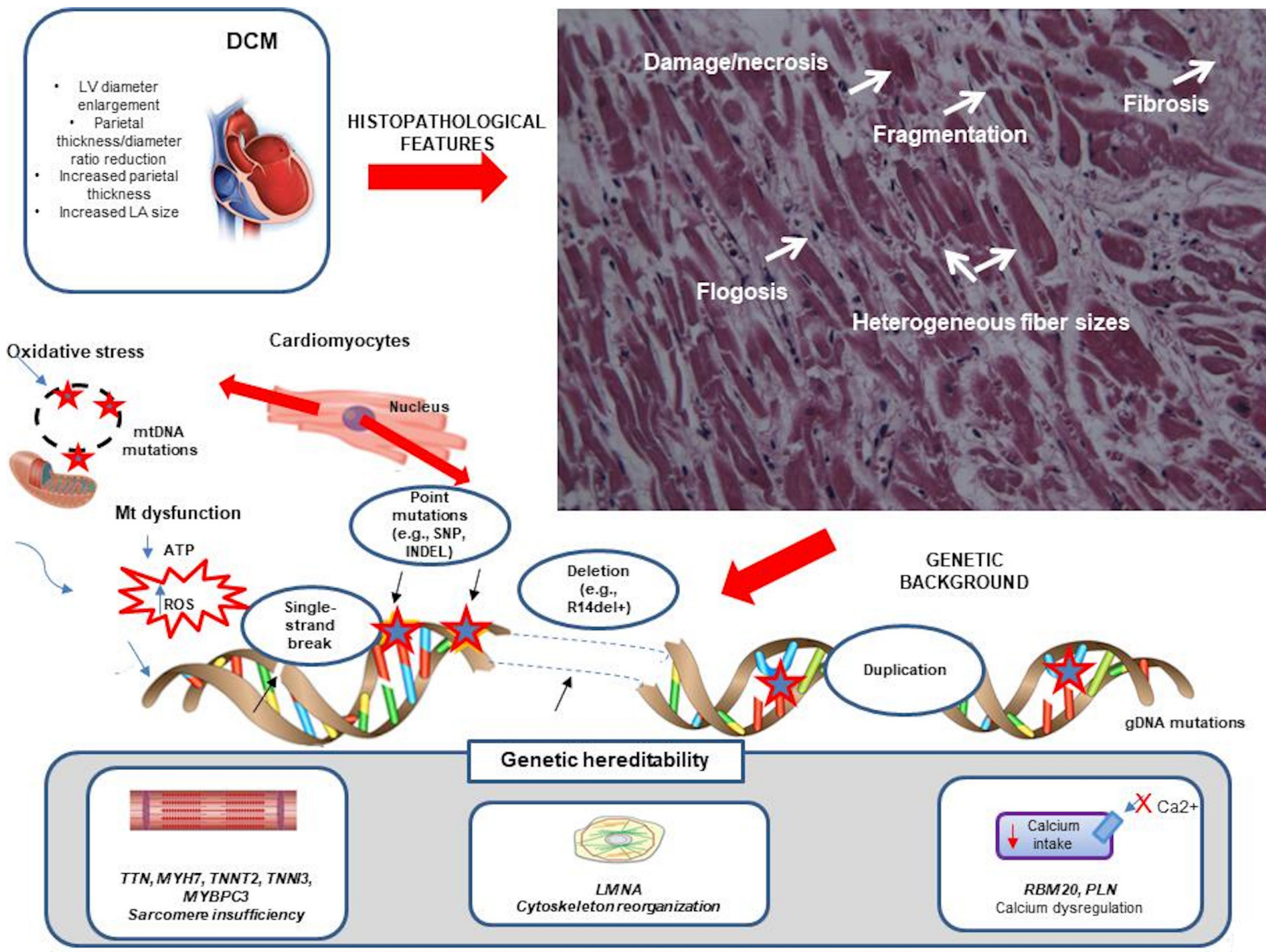

Figure 1 Myocardial injury and genetic of dilated cardiomyopathy (DCM). At the top, we illustrate structural and functional cardiac injury characterising patients with DCM with a focus on specific histopathological features by H\&E staining, including fiber fragmentation, interstitial fibrosis, damage and necrosis of cardiomyocytes, and heterogeneous fibre sizes (hypertrophy and atrophy) indicated by white arrows. At the bottom, we indicate heterogeneous chromosomal rearrangements, such as deletions/duplications, single-strand ruptures, as well as point mutations leading to specific abnormalities in eight DCM-causing genes: TTN, MYH7, TNNT2, TNNI3 and MYBPC3 involved in sarcomere insufficiency, LMNA correlated to cytoskeleton reorganisation, as well as RBM20 and PLN linked to calcium dysregulation. Moreover, we highlight the crucial role of oxidative stress for damaging mtDNA leading to decreased ATP and increased ROS, which contribute to increase the mutation rate of gDNA. gDNA, genomic DNA; LMNA lamin A/C; LV, left ventricular; mtDNA, mitochondrial DNA; MYBPC3, myosin binding protein C3; MYH7, myosin heavy chain 7; PLN, phospholamban; RBM20, RNA binding motif protein 20; ROS, reactive oxygen species; SNP, single nucleotide polymorphism; TTN, titin; TNNI3, troponin I3, cardiac type; TNNT2, troponin T2, cardiac type.

LV recovery and myocardial remodelling leading to advanced HF in order to better stratify different clinical phenotypes and improve personalised therapy (figures 2-4).

\section{CURRENT CHALLENGES IN DILATED CARDIOMYOPATHY}

DCM affects about 1 in 250 individuals and is classified in genetic, idiopathic, primitive or secondary disease, whose degree of myocardial dysfunction is not explained from an overload (table 1). ${ }^{6}$ Familial DCM is defined by the presence of two or more relatives with DCM or a history of SCD in an unexplained family, in which subjects may be classified as affected, unaffected or unknown (when subtle cardiac abnormalities are present but not sufficient for a definitive diagnosis). ${ }^{16}$ Both M-mode and two-dimensional echocardiography are frequently used to determine LV internal dimensions in systole (LVIDs) and diastole (LVIDd) ${ }^{17}$ Originally, it was thought that LV dilation occurs in response to reduced function. However, increased LV dimensions may already arise before a detectable reduction in $\mathrm{LV}$ function. ${ }^{18}$ Generally, LV dimensions are not the first detectable differences in familial DCM, whereas small LV dimensions were the first findings in HCM. ${ }^{18}$ Cardiac MRI accurately measures
LV chamber dimensions and function, including strain. ${ }^{19} 20$ The gadolinium and other contrast agents are used to evaluate fibrosis and therefore provide additional information on myocardial tissue quality. ${ }^{20}$ The delayed gadolinium enhancement may also reflect features beyond fibrosis including oedema and inflammatory infiltrate and is a predictor of all-cause mortality; specifically, delayed enhancement is associated with increased risk for ventricular arrhythmias. ${ }^{19}$ Endomyocardial biopsy (EMB) has been used to confirm specific and definite forms of DCM, but the complication rate with tissue biopsy ranges from $1 \%$ to $3 \%$ including serious complications such as perforation and tamponade occurring at $0.5 \%{ }^{18}$ For the majority of inherited cardiomyopathies, genetic testing is favoured over EMB. However, EMB can be useful to identify the underlying causes of primitive and/or idiopathic forms. It must be taken into consideration that DCM is one of the main indications for heart transplantation (HTx) which currently represents the only therapy for advanced states of disease. Moreover, EMB still plays a role in this field but certainly has a relevant role in post-transplant monitoring. ${ }^{11}$ DCM is regarded not as a single disease entity, but rather as heterogeneous phenotype with a final common 


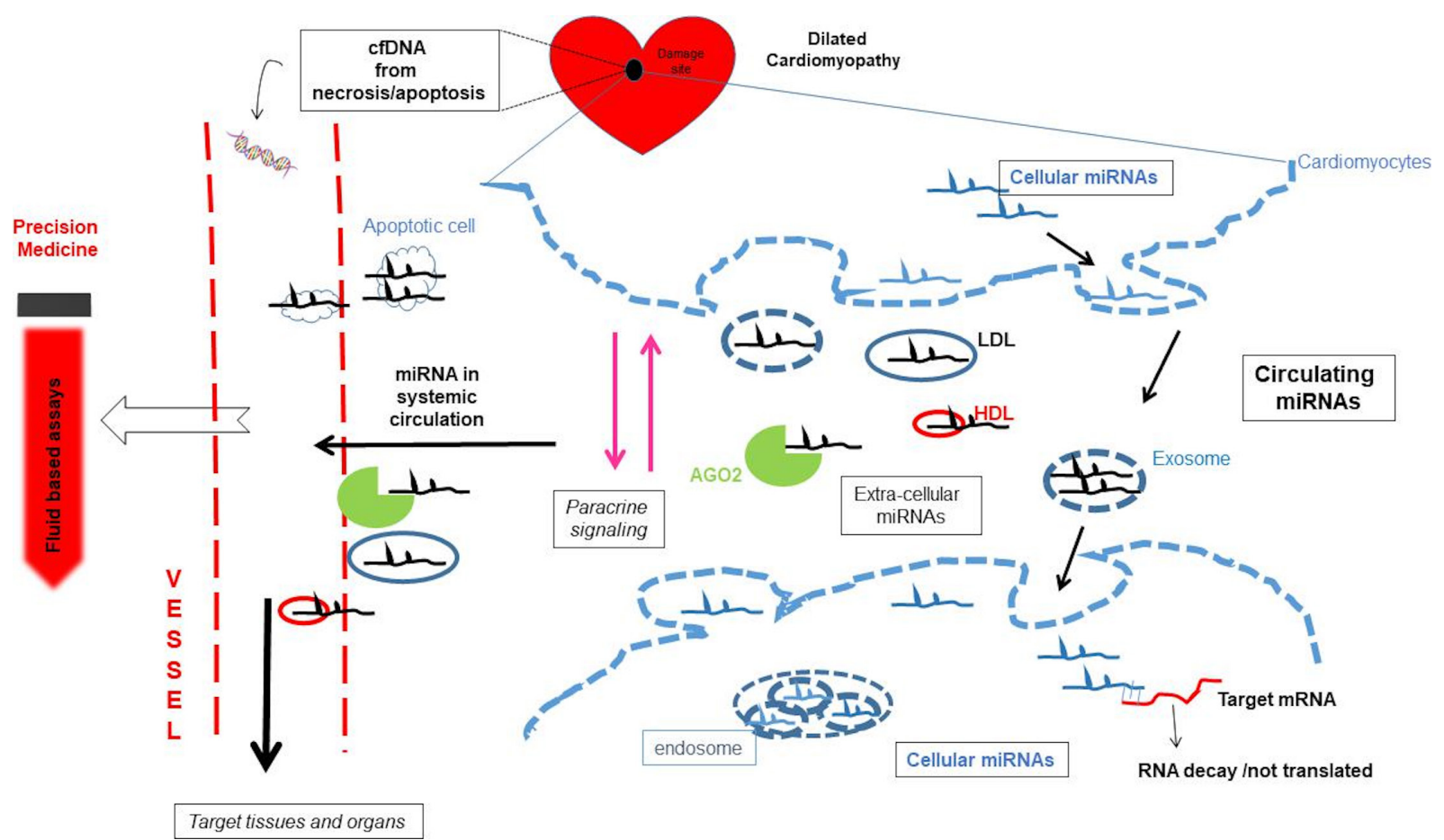

Figure 2 Apoptosis, cfDNA and miRNAs in dilated cardiomyopathy (DCM) precision medicine. In patients with DCM, failing hearts show apoptotic/ necrotic foci in which epigenetic-sensitive mechanisms can regulate cardiac remodelling, LV recovery and end-stage HF. In detail, circulating cfDNA are released in circulation from damage sites, reflecting the extent of cardiac damage. Moreover, specific cellular miRNAs are enveloped in microvesicles and exosomes and carried by LDL, HDL and AGO2 complex becoming circulating miRNAs able to regulate cell-cell communication and progression of disease. Current liquid-based assays can detect circulating molecular changes, providing novel perspectives in precision medicine of $\mathrm{HF}(\mathrm{see}$ the text for details). AGO2, argonaute family protein 2; cfDNA, cell-free DNA; HDL, high-density lipoprotein; HF, heart failure; LDL, low-density lipoprotein; LV, left ventricle; miRNAs, microRNAs.

response of the myocardium to a number of genetic and environmentally acquired insults. Common therapies and devices, such as $\beta$-blockers, renin-angiotensin-aldosterone inhibitors and cardiac resynchronisation therapy (CRT), can induce ' $\mathrm{LV}$ reverse remodelling', characterised by a decrease in LV volume and improved systolic function in patients with DCM. ${ }^{6}$ However, the knowledge regarding the exact stages and molecular changes for most causes of LV remodelling is quite limited. ${ }^{19}$ In particular, myocardial reverse remodelling has been reported in almost $40 \%$ of DCM patients with HFpEF.' In contrast, 'myocardial recovery' refers to normalisation of molecular, cellular, myocardial and LV geometric changes leading to preserved LV structure or function, without future HF events. ${ }^{6}$ Despite myocardial recovery has been frequently observed after LV assist device (LVAD) implantation, a functional and stable recovery of the heart has been observed less frequently leading to HTx after device removal in most of the patients. Remarkably, LV reverse remodelling does not mean molecular myocardial recovery; however, they may share similar downstream pathophysiological mechanisms increasing individual variability related to the end-stage phenotype of cardiac dilation and systolic dysfunction. $^{6}$ The identification of the first triggers and complex molecular mechanisms underlying DCM and its progression is fundamental for a timely and targeted therapeutic intervention in order to prevent the insurgence of HF. Many clinical studies reported a reduction in mortality rate with an increase in LVEF and a decrease in diastolic-endocentric LF volume which often occur in the presence of drug or device therapy. However, to date, it is not possible to predict who among the patients may show the capacity for cardiac recovery. ${ }^{6}$ Thus, novel circulating biomarkers may be useful to discriminate patients who maintain the potential for recovery of cardiac function in the clinical setting.

\section{GENETIC PLAYERS IN AETIOLOGY OF DCM}

Familial DCM is associated with more than 50 genes involved in the pathogenesis of $\mathrm{HF}$, each altering a targeted protein-protein interaction (PPI) in the human interactome, leading to abnormalities in different cellular activities; however, little is known about the precise mechanistic link between genotype and phenotype. ${ }^{21}$ By using NGS platforms, several DCM-causing variants were correlated with clinical and cardiac phenotypes as well as outcomes in patients and their relatives. Recently, a large metaanalysis of more than 8000 patients revealed that mutations in eight genes, including lamin $\mathrm{A} / \mathrm{C}$ (LMNA), phospholamban (PLN), RNA binding motif protein 20 (RBM20), myosin binding protein C3 (MYBPC3), pairs may heavy chain 7 (MYH7), troponin T2, cardiac type (TNNT2), troponin I3, cardiac type (TNNI3) and titin $(T T N)$ genes, show a predominant effect for phenotypic features in patients with DCM (figure 1). Moreover, a high prevalence of HTx and ventricular arrhythmia in carriers of both LMNA and PLN mutations were observed. ${ }^{22}$ Titin-truncating variants (TTNtvs) are the most common genetic cause of DCM, accounting for approximately $15 \%$ of cases. ${ }^{23} \mathrm{~A}$ recent study showed that TTNtvs are linked to eccentric cardiac remodelling, suggesting a role as 'at-risk' alleles in the general population. ${ }^{24}$ LMNA gene encodes for an ubiquitous protein expressed in the nuclear inner membrane involved in nuclear stability, chromatin structure and regulation of gene expression. ${ }^{25}$ The second most 


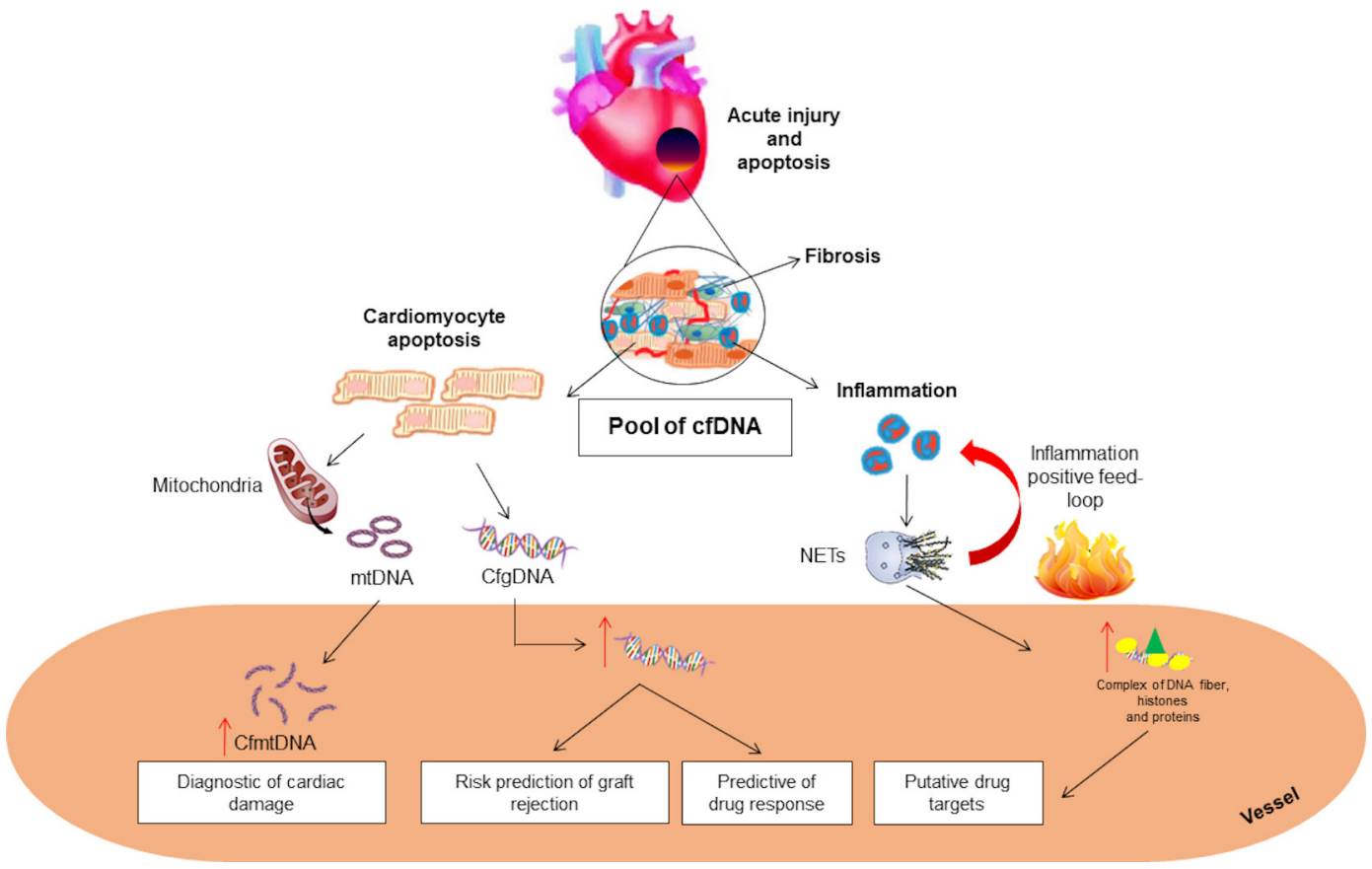

Figure 3 Putative role of cfDNA in precision medicine of advanced HF. Circulating cfDNA arise from acute injury and apoptosis in failing hearts. Increased levels of cfDNA can be detected in circulation of patients with HF, forming a heterogeneous pool of fragmented nucleotides. In detail, assessment of both gDNA and mtDNA may be useful biomarkers to diagnose cardiac damage and stratify the risk of early graft injury in patients with HF. Moreover, apoptotic neutrophil-released nets, formed by fibres of DNA combined with histones and proteins of granules, may represent a novel anti-inflammatory drug target in HF management. cfDNA, cell-free DNA; gDNA, genomic DNA; HF, heart failure; mtDNA: mitochondrial DNA; NETs, neutrophils extracellular traps.

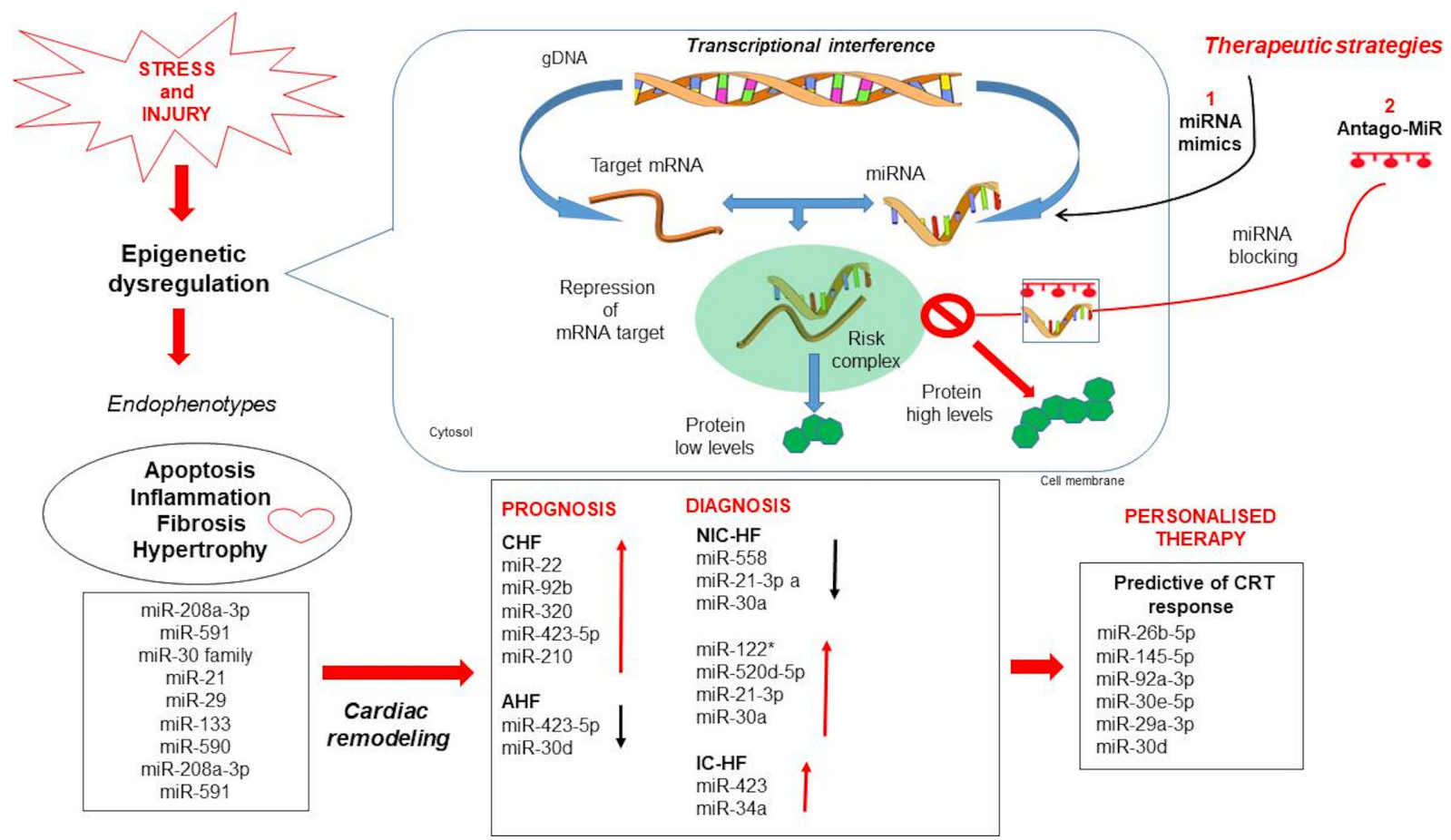

Figure 4 Basic mechanisms of miRNAs and their putative clinical use in advanced HF. At molecular level, miRNAs can associate with target mRNAs in the RISC complex, thus able to modulate gene expression at transcriptional level. Inhibitors (anti-miRNAs) of pathological miRNAs can modulate miRNA function, leading to an increase in expression of miRNA targets and beneficial effects. In contrast, administration of miRNA mimics can increase the repressive influence of beneficial miRNAs on their targets. We illustrate some putative panels of miRNAs useful to improve precision medicine and personalised therapy of HF. CRT, cardiac resynchronisation therapy; gDNA, genomic DNA; HF, heart failure; miRNAs, microRNAs; RISC, RNA-induced silencing complex. 


\begin{tabular}{|c|c|}
\hline \multicolumn{2}{|c|}{ Aetiology of dilated cardiomyopathy } \\
\hline \multirow[t]{3}{*}{ Primary } & Genetic \\
\hline & Non-genetic \\
\hline & Mixed \\
\hline \multirow[t]{38}{*}{ Secondary } & Myocardial disease \\
\hline & Coronary heart disease \\
\hline & Hypertension \\
\hline & Cardiomyopathy \\
\hline & Myocarditis \\
\hline & Endocardial disease \\
\hline & Endocarditis \\
\hline & Endocardial fibroelastosis \\
\hline & Pericardial disease \\
\hline & Constrictive pericarditis \\
\hline & Pericardial effusion \\
\hline & Arrhythmia and conduction disorders \\
\hline & Tachyarrhythmia atrial, ventricular \\
\hline & Bradyarrhythmia \\
\hline & Sinus node dysfunction \\
\hline & Atrioventricular block \\
\hline & Valvular heart disease \\
\hline & Mitral \\
\hline & Aortic \\
\hline & Tricuspid \\
\hline & Pulmonary \\
\hline & High output states \\
\hline & Anaemia \\
\hline & Thyrotoxicosis \\
\hline & Infection/Sepsis \\
\hline & Infiltrative/proliferative disease \\
\hline & Immunologic disease \\
\hline & Metabolic disease \\
\hline & Storage disease \\
\hline & Arteriovenous fistula \\
\hline & Volume overload \\
\hline & Renal failure \\
\hline & latrogenic (postoperative fluid infusion \\
\hline & Congenital heart disease \\
\hline & Stress-induced \\
\hline & Peripartum \\
\hline & Toxic origin \\
\hline & Other rare forms \\
\hline
\end{tabular}

Bold text represents subclassification of primary and secondary DCM. DCM, dilated cardiomyopathy.

commonly mutated gene is $L M N A$, encoding nuclear lamin A and $\mathrm{C}$ proteins. ${ }^{26}$ Additional mutations in the PLN, RBM20, MYH7, TNNT2 genes are responsible for architectural variations in myocardial structure and related dysfunction. ${ }^{22}$ Despite PLN mutations were directly associated with DCM onset and HF, many interactions with modifier genes may influence the clinical outcome. ${ }^{18}$ Moreover, it is estimated that dominant mutations in the RBM20 gene can contribute at least to $3 \%$ of idiopathic DCM cases. ${ }^{27}$ Interestingly, carriers of pathogenic RBM20 mutations have more ventricular arrhythmias than patients with TTN mutation, despite similarly reduced LV function, suggesting the need of a strict follow-up for potential electric disturbances and cardiac arrhythmias in the early stages of the disease. ${ }^{27} \mathrm{~A}$ crucial role in DCM onset and progression of HF is also played by mitochondrial DNA (mtDNA) damage and deletions, which may arise from radical oxygen species (ROS) mutating agents coupled with abnormalities in the peroxisome proliferatoractivated receptor $\gamma$ coactivator $1 \alpha(P G C-1 \alpha)$ gene activity. ${ }^{28}$ This molecular pathway reflects a maladaptive compensatory response contributing to increased oxidative stress leading to reduced ATP production and increased cell death, as hallmark of the LV failure. ${ }^{28}$ In table 2, we summarised the main candidate genes and related abnormalities associated with onset and development of familial DCM.

\section{THE ROLE OF CELL-FREE NUCLEIC ACIDS IN PRECISION MEDICINE AND PERSONALISED THERAPY OF ADVANCED HF}

Currently, some molecular biomarkers such as B-type natriuretic peptide (BNP) and $\mathrm{N}$-terminal pro-B-type natriuretic peptide (NT-proBNP), galectina-3, creatine kinase-MB (CK-MB) or cardiac troponins (cTn) $\mathrm{T}$ and I reflect apoptosis, myofibroblast proliferation, fibrosis, tissue repair, inflammation and LV remodelling associated with HF development representing established diagnostic and risk stratification tools. ${ }^{1529}$ Now, troponin detection shows an increased sensitivity and accuracy. In detail, the 99th percentile cut-point for the newest high-sensitivity TnT assay is $0.014 \mathrm{ng} / \mathrm{mL}$, which is very similar to the $0.01 \mathrm{ng} / \mathrm{mL}$ cut-point for the prior fourth generation TnT assay. ${ }^{30}$ However, there are still some limits in order to identify principal clinical events. Indeed, there are several causes of cardiac damage leading to troponin release that can be framed in a clinical context; furthermore, it should be taken into account that a slight basal increase can be present even in non-pathological conditions, as a response to physiological mechanisms. ${ }^{31}$ Therefore, there is a need for clarifying the mechanistic links by which novel biomarkers may (1) reflect progressive stages in HF onset, (2) establish cardio-specific panels and (3) discriminate patients able to recover LV function and benefit from personalised therapies.

We update on the possible role of circulating biomarkers to stratify the natural history of HF with much emphasis on cfDNA and miRNAs (table 3), which are strongly involved in regulating apoptosis and necrosis of myocardium, and discuss the putative clinical role of such liquid-based assays in management of patients with HF.

\section{Cell-free DNA}

The activation of different pathogenic molecular pathways triggered by the complex gene-environment relationship is implicated in the cardiac morphology and function instability by regulating the priming of key endophenotypes, such as apoptosis, inflammation and fibrosis leading to HF and SCD onset. ${ }^{52}$ Thus, a targeted pharmacological intervention aimed at modulating this complex chain of molecular perturbations may limit or retard cardiomyocyte damage. HF is strongly associated with cell death of both cardiac myocytes and endothelial cells. ${ }^{33}$ Circulating cfDNA mainly originates from acute cellular injury or apoptotic cells and substantially contributes to inflammatory responses. ${ }^{34}$ Physiologically, low concentrations of cfDNA $(2.5-27.0 \mathrm{ng} / \mathrm{mL})$ circulate in the serum/plasma from healthy individuals before being cleared by the liver. ${ }^{35}$ In contrast, increasing concentrations have been correlated widely to the extent of cellular damage in patients with $\mathrm{HF}^{11} \mathrm{~A}$ study reported that the unmethylated refilin A (FAM101A) gene may specifically trace cfDNA derived from cardiomyocyte death, providing a useful platform to measure apoptosis during ischaemia, complementing currently available biomarkers. ${ }^{36}$ Moreover, cfDNA plasma levels were significantly reduced in 19 
Table 2 Main DCM-causing genes

\begin{tabular}{|c|c|c|c|}
\hline Gene & Mutation class & Functional effect & Phenotypic outcome \\
\hline LMNA & $\begin{array}{l}\text { Indel, truncating, aberrant } \\
\text { splicing }\end{array}$ & GOF & $\begin{array}{l}\text { More aggressive clinical decline culminating in SCD owing to malignant ventricular arrhythmias and } \\
\text { end-stage HF. }\end{array}$ \\
\hline MYBPC3 & Indel, SNP, missense variants & GOF & $\begin{array}{l}\text { Contractile deficiencies leading to severe mechanical stress and exacerbated cardiac myocyte } \\
\text { apoptosis and necrosis inducing myocardial inflammation and severity of disease. }\end{array}$ \\
\hline MYH7 & SNP & LOF & Reduced contractility resulting from impaired sarcomere function. \\
\hline$P L N$ & $\begin{array}{l}\text { Deletion } \\
\text { (R14del+) }\end{array}$ & LOF & $\begin{array}{l}\text { Reduced } \mathrm{Ca}^{+} \text {uptake in sarcolemma, desmosomal disassembly and increased cytoplasmic } \mathrm{Ca}^{+} \\
\text {levels correlated with the presence of malignant ventricular arrhythmias and interstitial fibrosis. }\end{array}$ \\
\hline RBM20 & SNP, missense variant, frameshift & LOF & $\begin{array}{l}\text { Impaired nuclear localisation of RBM20 protein leading to aberrant cytoskeleton assembly correlated } \\
\text { with systolic dysfunction, LV dilatation and higher risk of ventricular arrhythmia. }\end{array}$ \\
\hline TTN & $\begin{array}{l}\text { SNP, Indel, frameshift, truncating, } \\
\text { aberrant splicing }\end{array}$ & $\begin{array}{l}\text { Haploinsufficiency, } \\
\text { dominant negative }\end{array}$ & Disruption of sarcomere structure leading to cardiac dysfunction and remodelling. \\
\hline TNNT2 & Indel, SNP & LOF & Myofilament dysfunction mediated by significant increase in passive tension. \\
\hline TNNI3 & SNP, truncating & $\begin{array}{l}\text { Haploinsufficiency, } \\
\text { dominant negative }\end{array}$ & $\begin{array}{l}\text { Myofilament dysfunction owing to increased } \mathrm{Ca}_{2}^{+} \text {sensitivity and impaired length-dependent } \\
\text { activation. }\end{array}$ \\
\hline
\end{tabular}

$A D$, autosomal dominant; DCM, dilated cardiomyopathy; GOF, gain of function; HF, heart failure; Indel, insertion and deletion; LMNA, lamin A/C; LOF, loss of function; MYBPC3, myosin-binding protein C3; MYH7, myosin-heavy chain 7; PLN, phospholamban; RBM20, RNA binding motif 20; SCD, sudden cardiac death; SNP, single nucleotide polymorphism; TNNI3, troponin I3 cardiac type; TNNT2, troponin T2 cardiac type; TTN, titin.

ischaemic HF patients with severe LV systolic dysfunction after 12 hours from levosimendan infusion with respect to controls (not treated patients) and correlated with improvement of echocardiographic and biochemical indices of LV function, suggesting a surrogate biomarker of myocardial performance. ${ }^{37}$ Despite LVADs may reduce apoptosis, a pilot study demonstrated that pretransplant LVAD implantation is correlated with higher risk of early graft injury evaluated by detection of increased levels of

Table 3 Examples of clinical studies evaluating cfDNA and miRNAs in advanced HF

\begin{tabular}{|c|c|c|c|c|c|}
\hline Probe & Sample size & Sample source & Platform & Results & Refs. \\
\hline $\begin{array}{l}\text { Unmethylated } \\
\text { FAM101A }\end{array}$ & $\begin{array}{l}74 \text { STEMI patients versus } 83 \text { healthy } \\
\text { subjects }\end{array}$ & Plasma & Bisulfite sequencing & $\begin{array}{l}\text { Higher levels of cfDNA were strongly associated } \\
\text { with STEMI patients }\end{array}$ & 36 \\
\hline cfDNA & $\begin{array}{l}19 \text { ischaemic HF patients before and } \\
12 \text { hours after levosimendan infusion }\end{array}$ & Plasma & qPCR & $\begin{array}{l}\text { cfDNA was significantly reduced in HF patients } \\
\text { post-levosimendan treatment }\end{array}$ & 37 \\
\hline mtDNA & $\begin{array}{l}90 \text { acute HF versus } 109 \text { chronic HF } \\
\text { patients }\end{array}$ & Plasma & qPCR & $\begin{array}{l}\text { Higher levels of mtDNA were associated with } \\
\text { mortality rate in patients with severe acute HF }\end{array}$ & 39 \\
\hline miRNAs & $\begin{array}{l}30 \text { stable chronic HF patients versus } \\
30 \text { healthy controls }\end{array}$ & Serum & qPCR & $\begin{array}{l}\text { Higher levels of miR-423-5p, miR-320a, miR- } \\
22 \text { and miR-92b may be useful diagnostic } \\
\text { biomarker of chronic HF }\end{array}$ & 43 \\
\hline miRNAs & $\begin{array}{l}236 \text { acute } \mathrm{HF} \text { and } 58 \text { non-acute HF } \\
\text { versus } 44 \text { stable chronic HF }\end{array}$ & Plasma & $\mathrm{qPCR}$ & $\begin{array}{l}\text { Baseline low levels of miR-423-5p were } \\
\text { associated with a poor prognosis in acute HF }\end{array}$ & 44 \\
\hline miRNAs & $\begin{array}{l}96 \text { acute HF patients followed up } \\
\text { for } 1 \text { year }\end{array}$ & Serum & qPCR & $\begin{array}{l}\text { Lower miR-30d levels were strongly predictive } \\
\text { of mortality in acute HF patients }\end{array}$ & 45 \\
\hline miRNAs & $\begin{array}{l}13 \text { patients with congestive HF } \\
\text { versus six healthy controls }\end{array}$ & $\begin{array}{l}\text { Circulating } \\
\text { mononuclear cells }\end{array}$ & qPCR & $\begin{array}{l}\text { miR-210 levels were upregulated in severe } \\
\text { NYHA III and IV versus NYHA II HF patients }\end{array}$ & 46 \\
\hline miRNAs & $\begin{array}{l}39 \mathrm{HFrEF} \text { and } 19 \mathrm{HFpEF} \text { patients } \\
\text { versus } 28 \text { healthy controls }\end{array}$ & Plasma & miRCURY LNA microRNA Arrays & $\begin{array}{l}\text { Upregulation of miR-125a-5p, miR-550a- } 5 p \text {, } \\
\text { miR-638 and downregulation of miR-190a may } \\
\text { discriminate HFrEF from HFpEF }\end{array}$ & 48 \\
\hline miRNAs & $\begin{array}{l}90 \mathrm{HFrEF} \text { and } 90 \mathrm{HFpEF} \text { versus } 90 \\
\text { no-HF patients (control group) }\end{array}$ & Plasma & Taqman miRNA arrays & $\begin{array}{l}\text { Differential expression of miR-328, miR-30c, } \\
\text { miR-221 and miR-375 could discriminate HFpEF } \\
\text { and HFrEF }\end{array}$ & 49 \\
\hline miRNAs & $\begin{array}{l}23 \mathrm{NICM}-\mathrm{HF} \text { and } 41 \text { ICM-HF versus } \\
11 \text { healthy controls }\end{array}$ & Plasma & qPCR & $\begin{array}{l}\text { Upregulation ofmiR- } 423 / \mathrm{miR}-34 \mathrm{a} \text { and miR- } \\
21-3 \mathrm{p} / \mathrm{miR}-30 \text { a pairs may discriminate ICM-HF } \\
\text { versus NICM-HF, respectively }\end{array}$ & 8 \\
\hline miRNAs & $\begin{array}{l}81 \mathrm{HF} \text { patients undergoing CRT } \\
\text { versus } 15 \text { healthy controls }\end{array}$ & Serum & qPCR & $\begin{array}{l}\text { Upregulation ofmiR-26b-5p, miR-145-5p, } \\
\text { miR-92a-3p, miR-30e-5p and miR-29a-3p } \\
\text { was correlated to reverse remodelling in CRT } \\
\text { responders }\end{array}$ & 53 \\
\hline
\end{tabular}

cfDNA, cell-free DNA; CRT, cardiac resynchronisation therapy; FAM101A, Refilin A; HF, heart failure; HFpEF, heart failure preserved ejection fraction; HFrEF, heart failure reduced ejection fraction; ICM-HF, ischaemic HF; miRNAs, microRNAs; mtDNA, mitochondrial DNA; NICM-HF, non-ischaemic HF; NYHA, New York Heart Association; qPCR, quantitative PCR; STEMI, ST-elevation myocardial infarction. 
donor-derived cfDNA (dd-cfDNA) in the plasma of recipients. ${ }^{38}$ Recently, elevated cell-free mtDNA levels have been associated with high mortality rate in patients with severe acute HF rather than in chronic disease phenotype, suggesting a useful biomarker to improve HF risk stratification. ${ }^{39}$ Now, the use of cfDNA is of limited clinical utility and warrants further research. In figure 3, we illustrate the putative role of cfDNA as clinical biomarkers in patients with HF.

\section{miRNAs}

miRNAs are single-stranded small non-coding RNAs (20-24 nucleotide) originated from a stem-loop precursor in the nucleus and matured in the cytoplasm where they regulate a majority of protein-coding genes in health and disease. ${ }^{40}$ miRNAs can regulate gene expression at the post-transcriptional level by the recognition of complementary region in the $3^{\prime}$ untranslated region ( 3 '-UTR) of many mRNA targets in order to determine the degradation or repression of the translation (figure 4). Moreover, miRNAs can be transported outside the cells and exosomes, microvesicles, apoptotic bodies, RNA binding proteins such as nucleophosmin (NPM1) and the argonaute family proteins (Ago $2,1,3,4)$; high-density lipoprotein (HDL) and low-density lipoprotein (LDL) represent heterogeneous carriers to transfer functional information (figure 2). ${ }^{41}$ The implementation of miRNAs in clinical use may challenge diagnosis and risk stratification of patients with HF. miRNAs also offer great therapeutic opportunities by administration of oligonucleotide inhibitors (anti-miRs) and miRNA mimics (figure 4). In detail, anti-miRs are designed to directly target specific disease-associated miRNA resulting in the upregulation of protein expression; they are efficiently taken up by a variety of tissues following systemic delivery and are well tolerated without toxicity. Conversely, miRNA mimics can enhance the repressive miRNA function on their targets resulting in protein downregulation (figure 4) suggesting a useful strategy to diminish disease progression. By using liquid-based assays, aberrant miRNA expression profiles have been correlated to HF onset and allograft rejection, suggesting putative useful diagnostic and prognostic biomarkers. ${ }^{8} 1142 \mathrm{~A}$ strong correlation between miR-423-5 p, miR-320a, miR-22 and miR-92b upregulation and clinical prognostic parameters, including elevated natriuretic peptide levels, a wide QRS and LV and LA, in 30 chronic systolic HF patients versus controls was observed, suggesting these molecules as added value in improving current disease prognosis. ${ }^{43}$ Recently, low plasma levels of miR-423-5 p were detected in acute HF with respect to non-acute and stable chronic HF patients showing a predictive value for 1-year readmission to the hospital, suggesting a useful non-invasive prognostic biomarker. ${ }^{44}$ Another prospective study has suggested the putative use of serum miR-30d low levels as predictive biomarker of 1-year all-cause mortality in patients with acute $\mathrm{HF}^{45}$ Furthermore, increased levels of hypoxiainduced miR-210 plasma levels were detected at baseline in mononuclear cells isolated from 39 patients with advanced HF (NYHA III and IV class) with respect to non-advanced HF (NYHA II class) and controls. ${ }^{46}$ In addition, high plasma miR-210 levels correlated with plasma BNP concentrations at the subsequent hospital visit about 3 months late, demonstrating a putative useful role in predicting poor prognosis of patients with severe $\mathrm{HF}^{46}$ For the first time, some authors investigated the possible diagnostic role of miRNAs both in plasma and some peripheral blood cells to discriminate non-ischaemic HFrEF $(n=53)$ versus healthy controls $(n=39) .{ }^{47}$ Results from this study reported a strong correlation between downregulation of
miR-558 and upregulation of miR-122* and miR-520d-5p with HFrEF patients suggesting their usefulness in discriminating HF when used as single indicators. ${ }^{47}$ Remarkably, two independent studies identified a panel of miRNAs able to discriminate HFrEF from HFpEF patients. ${ }^{48} 49$ By examining a large study cohort $(\mathrm{n}=176)$, Wong et $a l^{48}$ reported that upregulation of miR$125 a-5 p$, miR-550a-5p, miR-638 as well as downregulation of miR-190a may strongly distinguish HFrEF from HFpEF patients with a major discriminative power than NT-proBNP suggesting a useful panel for differential diagnosis. In a more large cohort $(n=225)$, it was reported that the diagnostic value of BNP could be improved by combining a panel of four miRNAs: miR-328, miR-30c, miR-221 and miR-375. ${ }^{49}$ In detail, miR-328 was upregulated, whereas miR-375, miR-221 and miR-30c were downregulated in HFrEF with respect to HFpEF. ${ }^{49}$ Moreover, miRNA levels have been measured across the coronary circulation in 75 subjects undergoing coronary angiography at the catheterisation to assess their clinical utility in discriminating different HF subgroups, including ICM-HF, NICM-HF, ICM$\mathrm{HF}+\mathrm{NICM}-\mathrm{HF}$ with respect to controls. ${ }^{8}$ By results, a positive transcoronary gradient was found for upregulated miR-423 and miR-34a in the ICM-HF group and upregulated miR-21-3 $p$ and miR-30a in the NICM-HF group suggesting these molecules as useful cardiac-specific biomarkers to discriminate different HF phenotypes. ${ }^{8}$ Moreover, Sucharov et $a l^{50}$ demonstrated that downregulation of miR-208a-3p, miR-591, miR-208a-3p, miR$208 b-3 p$, miR-21-5 p and 199a-5p, as well as upregulation of miR-1-3 $p$ in bioptic tissues isolated from DCM and IDC patients, may be predictive of favourable reverse remodelling in response to $\beta$-blocker treatment at 3 or 12 months with respect to 'nonresponders' (NCT01798992). Thus, these intracellular miRNAs should be investigated in plasma/serum samples, also. Interestingly, pathway analysis reported that selected miRNA-mRNA interactions were predictive of improved cardiac contractility, decreased myocardial hypertrophy apoptosis necrosis. ${ }^{50}$ The inverse remodelling occurs in the early stages of HF with patients undergoing neurohormonal therapy, CRT cardiac resynchronisation, revascularisation and elimination of precipitating factors. ${ }^{51}$ Identifying molecular pathways leading to reverse remodelling is essential to develop novel therapies useful in early phases of HF onset in order to delay disease progression, repair structure and function of myocytes and promote myocardial recovery. This would limit progression to advanced HF and HTx by improving stratification of candidates to HTx or LVAD implantation. ${ }^{51} 52$ Interestingly, Marfella et $a l^{53}$ found higher baseline levels of miR-26b-5p, miR-145-5 p, miR-92a-3p, miR-30e-5p and miR$29 a-3 p$ in CRT responders with respect to non-responders, suggesting useful predictive biomarkers. Moreover, Melman et $a l^{54}$ demonstrated that increased baseline levels of miR-30d in plasma coronary sinus was a putative biomarker useful in discriminating responders versus non-responder patients to CRT pacemaker implantation, owing to a functional role in adaptive hypertrophy. In figure 4, we illustrated the basic miRNA mechanism of action and miRNA-based therapeutic strategies by highlighting their putative clinical role in personalised therapy of advanced HF.

\section{DISCUSSION}

Nowadays, the accurate classification of HF subtypes remains challenging but of crucial importance to stratify patients and customise drug therapies. ${ }^{1}$ Indeed, discriminating HFpEF from HFmEF and HFrEF would be clinically relevant but it it is not provided by the current established biomarkers. ${ }^{2055}$ Therefore, 
a detailed map of molecular networks perturbing the 'cardiac interactome' may reveal novel useful biomarkers or drug targets specific for each phenotype. Development of HF is preceded by cardiac remodelling in which there is a progressive enlargement of heart chambers and impairment of contractile function. Cardiomyocyte apoptosis represents a pivotal form of cell death in ischaemia and reperfusion damage showing a rate of $2 \%-12 \%$ in the border zone of human myocardial infarcts. ${ }^{56}$ Moreover, endothelial cell apoptosis is also often found in HF biopsies and could contribute to heart decompensation owing to alteration of blood perfusion (ischaemia, lack of oxygen, free radicals and so on). ${ }^{33}$ Since apoptosis is considered a key endophenotype during transition from compensatory hypertrophy to HF onset, both identification and quantification of cell death could be relevant for diagnosis, risk stratification and treatment of HF. To date, cardiac-specific troponins represent the gold standard for diagnosis of both ischaemic and non-ischaemic myocardial damage; however, the current challenge would be to identify a precise cut-off able to accurately discriminate physiological from non-physiological conditions (eg, physical exercise). ${ }^{31}$ Advanced NGS platforms and various fluid-based assays are increasing our knowledge about the mechanistic link between apoptosis and progressive stages of HF development, suggesting cell-free nucleic acids, mainly cfDNA (genomic DNA and mtDNA) and miRNAs, as putative non-invasive biomarkers. ${ }^{11}$ Now, detection of cfDNA levels, as hallmark of cell death, is emerging as a powerful diagnostic tool for myocardial performance and graft rejection. In particular, we emphasised the approach performed by Zemmour $e a^{36}{ }^{36}$ showing that unmethylated cfDNA (starting material from $0.5 \mathrm{~mL}$ of plasma) may be useful to estimate cardiac-specific cfDNA in a heterogeneous pool of circulating DNA molecules. Another form of cfDNA, enveloped in neutrophil extracellular traps (NETs), has been detected in cardiac tissue of patients with $\mathrm{HF}^{57}$ (figure 3). These molecular complexes are characterised by fibres of DNA combined with histones and proteins of granules, such as serine proteases, cathepsin $G$ and myeloperoxidase (MPO) which arise from apoptotic neutrophils on inflammatory stimuli. ${ }^{11}$ Thus, NETs may be novel drug targets in HF management. ${ }^{57}$ However, detection of NETs is less sensitive with respect to cfDNA; indeed, it was performed by using ELISA which measures exclusively MPO-bound DNA complexes in (starting material from $25 \mathrm{~mL}$ ) serum samples of patients with $\mathrm{HF}^{57}$ Remarkably, data regarding cfDNA are simply associative in nature and are unclear whether they are specific to HF, their specific causes or treatment responses. Indeed, the implication that they all originated from the failing heart is largely assumed especially in human studies. Therefore, further investigations are needed to improve specificity and sensitivity of current methodologies to detect different forms of cfDNA and corroborate its putative clinical role in patients with $\mathrm{HF}^{58}{ }^{59}$ Also, miRNA microarray profiling or quantitative PCR (qPCR) platforms have examined the miRNA profiles obtained from various HF cohorts (figure 4). However, miRNA investigation in HF have not been impressive owing, in part, to the heterogeneous variety of experimental conditions used by independent groups, for example, plasma versus serum samples and small study population, making comparisons and interpretation highly difficult. Based on the consistent study population $(n=225)$ investigated by Watson et $a l{ }^{49}$ we emphasised that combination of miR-328, miR-30c, miR-221 and miR-375 may be suitable for further observation to discriminate HFrEF with respect to HFpEF. The next step in the development of miRNA-based drugs would be to clarify both molecular pathways and cell targets involved in their biological functions in order to reduce putative side effects.
To date, the implementation of liquid-based assays detecting circulating cfDNA and miRNAs is far from the routine clinical use. ${ }^{60}$ Overall, some technical limitations of these markers, including biological, interassay/intra-assay or sampling variabilities, as well as the lack of insights into their sources or associations with circulating cells versus cardiac or non-cardiac tissues, are still unsolved issues. ${ }^{61}$ Liquid-based assays are promising diagnostic tools; however, their possible clinical role will be established when and whether the presence of circulating biomarkers will be also confirmed in disease-related with respect to normal tissues. Moreover, further challenges of these post hoc analysis on already indicated therapies ( $\beta$-blockers and CRT) are ongoing. Interestingly, some panels of specific circulating miRNAs may be useful in predicting responders versus nonresponders. ${ }^{5053}$

Therefore, clinical trials should be designed to overcome the current methodological barriers by using advanced networkoriented analysis in order to establish specific and accurate noninvasive biomarkers able to complement imaging techniques and cardiac troponins and BNP/NT-proBNP in detecting LV structural and functional abnormalities. ${ }^{62}$

\section{Take home messages}

- Advanced omics tools may provide liquid-based assays useful to reach precision medicine and personalized therapy of patients with advanced heart failure.

- Myocyte and endothelial cell apoptosis is key driver of cardiac remodeling.

- Circulating molecules may be useful biomarkers for HF personalised therapy

Handling editor Tahir S Pillay.

Contributors CN: genesis of idea. GM, GB, NDM: review of literature, design of tables and figures, initial draft of the manuscript. CN and GFN: critical and final review of the paper.

Funding This work was supported by PRIN2017F8ZB89 from Italian Ministry of University and Research (MIUR) (PI Professor Napoli). The funders had no role in study design, data collection and analysis, decision to publish or preparation of the manuscript. Dr Giuditta Benincasa is a PhD student of Translational Medicine awarded with Educational Grant from ESC Congress 2019 and supported by Educational Grant from the University of Campania, Naples, Italy.

Competing interests None declared.

Patient consent for publication Not required.

Provenance and peer review Not commissioned; externally peer reviewed.

\section{ORCID iDs}

Gelsomina Mansueto http://orcid.org/0000-0002-0544-5100

Giuditta Benincasa http://orcid.org/0000-0002-7552-3522

Nunzia Della Mura http://orcid.org/0000-0002-6383-2176

\section{REFERENCES}

1 Yancy CW, Jessup M, Bozkurt B, et al. 2017 ACC/AHA/HFSA Focused Update of the 2013 ACCF/AHA Guideline for the Management of Heart Failure: A Report of the American College of Cardiology/American Heart Association Task Force on Clinical Practice Guidelines and the Heart Failure Society of America. J Am Coll Cardiol 2017;70:776-803.

2 Crimi E, Ignarro LJ, Cacciatore $F$, et al. Mechanisms by which exercise training benefits patients with heart failure. Nat Rev Cardiol 2009;6:292-300.

3 Cacciatore F, Amarelli C, Ferrara N, et al. Protective effect of physical activity on mortality in older adults with advanced chronic heart failure: a prospective observational study. Eur J Prev Cardiol 2019;26:481-8.

4 Gronda E, Vanoli E, Sacchi S, et al. Risk of heart failure progression in patients with reduced ejection fraction: mechanisms and therapeutic options. Heart Fail Rev 2020;25:295-303

5 Gronda E, Sacchi S, Benincasa G, et al. Unresolved issues in left ventricular postischemic remodeling and progression to heart failure. J Cardiovasc Med 2019;20:640-9. 
6 Tayal U, Prasad SK. Myocardial remodelling and recovery in dilated cardiomyopathy. IRSM Cardiovasc Dis 2017;6:204800401773447.

7 Napoli C, Casamassimi A, Crudele V, et al. Kidney and heart interactions during cardiorenal syndrome: a molecular and clinical pathogenic framework. Future Cardiol 2011;7:485-97.

8 De Rosa S, Eposito F, Carella C, et al. Transcoronary concentration gradients of circulating microRNAs in heart failure. Eur J Heart Fail 2018;20:1000-10.

9 Napoli C, Benincasa G, Loscalzo J. Epigenetic inheritance underlying pulmonary arterial hypertension. Arterioscler Thromb Vasc Biol 2019;39:653-64.

10 Napoli C, Benincasa G, Schiano C, et al. Differential epigenetic factors in the prediction of cardiovascular risk in diabetic patients. Eur Heart J Cardiovasc Pharmacother 2019. doi:10.1093/ehjcvp/pvz062. [Epub ahead of print: 26 Oct 2019].

11 Benincasa G, Mansueto G, Napoli C. Fluid-based assays and precision medicine of cardiovascular diseases: the 'hope' for Pandora's box? J Clin Pathol 2019;72:785-99.

12 Benincasa G, Costa D, Infante T, et al. Interplay between genetics and epigenetics in modulating the risk of venous thromboembolism: a new challenge for personalized therapy. Thromb Res 2019;177:145-53.

13 D'Onofrio N, Sardu C, Paolisso P, et al. MicroRNA-33 and SIRT1 influence the coronary thrombus burden in hyperglycemic STEMI patients. I Cell Physiol 2020;235:1438-52.

14 Schiano C, Costa V, Aprile M, et al. Heart failure: pilot transcriptomic analysis of cardiac tissue by RNA-sequencing. Cardiol I 2017;24:539-53.

15 Schiano C, Vietri MT, Grimaldi V, et al. Epigenetic-related therapeutic challenges in cardiovascular disease. Trends Pharmacol Sci 2015;36:226-35.

16 Arbustini E, Narula N, Tavazzi L, et al. The MOGE(S) classification of cardiomyopathy for clinicians. J Am Coll Cardiol 2014;64:304-18.

17 Chengode $S$. Left ventricular global systolic function assessment by echocardiography. Ann Card Anaesth 2016;19:26-34.

18 McNally EM, Mestroni L. Dilated cardiomyopathy: genetic determinants and mechanisms. Circ Res 2017;121:731-48.

19 Cordero-Reyes AM, Youker K, Estep JD, et al. Molecular and cellular correlates of cardiac function in end-stage DCM: a study using speckle tracking echocardiography. JACC Cardiovasc Imaging 2014:7:441-52.

20 Infante T, Del Viscovo L, De Rimini ML, et al. Network medicine: a clinical approach for precision medicine and personalized therapy in coronary heart disease. J Atheroscler Thromb 2020:27:279-302.

21 van der Bijl P, Delgado V, Bootsma M, et al. Risk stratification of genetic, dilated cardiomyopathies associated with neuromuscular disorders: role of cardiac imaging. Circulation 2018;137:2514-27.

22 Kayvanpour E, Sedaghat-Hamedani F, Amr A, et al. Genotype-Phenotype associations in dilated cardiomyopathy: meta-analysis on more than 8000 individuals. Clin Res Cardiol 2017:106:127-39.

23 Corden B, Jarman J, Whiffin N, et al. Association of titin-truncating genetic variants with life-threatening cardiac arrhythmias in patients with dilated cardiomyopathy and implanted defibrillators. JAMA Netw Open 2019;2:e196520.

24 Schafer S, de Marvao A, Adami E, et al. Titin-truncating variants affect heart function in disease cohorts and the general population. Nat Genet 2017:49:46-53.

25 Gonzalo S. Dna damage and lamins. Adv Exp Med Biol 2014;773:377-99.

26 Nishiuchi S, Makiyama T, Aiba T, et al. Gene-Based Risk Stratification for Cardiac Disorders in LMNA Mutation Carriers. Circ Cardiovasc Genet 2017;10:10.

27 Wyles SP, Li X, Hrstka SC, et al. Modeling structural and functional deficiencies of RBM20 familial dilated cardiomyopathy using human induced pluripotent stem cells. Hum Mol Genet 2016;25:254-65.

28 Rohani L, Meng G, Machiraju P, et al. Modeling the dilated cardiomyopathy with ataxia syndrome (DCMA), a pediatric mitochondrial cardiomyopathy, using cardiomyocytes derived from induced pluripotent stem cells. Can J Cardiol 2017;33:S163-4.

29 Kavsak PA, Lam CSP, Saenger AK, et al. Educational recommendations on selected analytical and clinical aspects of natriuretic peptides with a focus on heart failure: a report from the IFCC Committee on clinical applications of cardiac bio-markers. Clin Chem 2019;65:1221-7

30 Brush JE, Kaul S, Krumholz HM. Troponin testing for clinicians. J Am Coll Cardiol 2016:68:2365-75.

31 Roca E, Nescolarde L, Lupón J, et al. The dynamics of cardiovascular biomarkers in non-elite marathon runners. J Cardiovasc Trans/ Res 2017:10:206-8.

32 Napoli C, Schiano C, Soricelli A. Increasing evidence of pathogenic role of the mediator (Med) complex in the development of cardiovascular diseases. Biochimie 2019;165:1-8.

33 Gogiraju R, Bochenek ML, Schäfer K. Angiogenic endothelial cell signaling in cardiac hypertrophy and heart failure. Front Cardiovasc Med 2019;6:20.
34 Marsman G, Zeerleder S, Luken BM. Extracellular histones, cell-free DNA, or nucleosomes: differences in immunostimulation. Cell Death Dis 2016;7:e2518.

35 Lou X, Hou Y, Liang D, et al. A novel Alu-based real-time PCR method for the quantitative detection of plasma circulating cell-free DNA: sensitivity and specificity for the diagnosis of myocardial infarction. Int J Mol Med 2015;35:72-80.

36 Zemmour H, Planer D, Magenheim J, et al. Non-Invasive detection of human cardiomyocyte death using methylation patterns of circulating DNA. Nat Commun 2018:9:1443.

37 Zaravinos A, Tzoras S, Apostolakis S, et al. Levosimendan reduces plasma cell-free DNA levels in patients with ischemic cardiomyopathy. J Thromb Thrombolysis 2011;31:180-7.

38 Rodrigo ME, Agbor-Enoh S, Gorham S, et al. Association between LVAD and cardiac allograft injury following heart transplantation as assessed by cell-free DNA. J Heart Lung Transplant 2017;36:S397.

39 Krychtiuk KA, Wurm R, Ruhittel $\mathrm{S}$, et al. Release of mitochondrial DNA is associated with mortality in severe acute heart failure. Eur Heart J Acute Cardiovasc Care 2019:204887261882340.

40 Holoch D, Moazed D. Rna-Mediated epigenetic regulation of gene expression. Nat Rev Genet 2015:16:71-84.

41 Rayner KJ, Hennessy EJ. Extracellular communication via microRNA: lipid particles have a new message. J Lipid Res 2013;54:1174-81.

42 Sabia C, Picascia A, Grimaldi V, et al. The epigenetic promise to improve prognosis of heart failure and heart transplantation. Transplant Rev 2017;31:249-56.

43 Goren Y, Kushnir M, Zafrir B, et al. Serum levels of microRNAs in patients with heart failure. Eur J Heart Fail 2012;14:147-54.

44 Seronde M-F, Vausort M, Gayat E, et al. Circulating microRNAs and outcome in patients with acute heart failure. PLoS One 2015;10:e0142237.

45 Xiao J, Gao R, Bei Y, et al. Circulating miR-30d predicts survival in patients with acute heart failure. Cell Physiol Biochem 2017;41:865-74.

46 Endo K, Naito Y, Ji X, et al. Microrna 210 as a biomarker for congestive heart failure. Biol Pharm Bull 2013;36:48-54.

47 Vogel B, Keller A, Frese KS, et al. Multivariate miRNA signatures as biomarkers for non-ischaemic systolic heart failure. Eur Heart J 2013;34:2812-23.

48 Wong LL, Armugam A, Sepramaniam S, et al. Circulating microRNAs in heart failure with reduced and preserved left ventricular ejection fraction. Eur I Heart Fail 2015;17:393-404

49 Watson CJ, Gupta SK, O'Connell E, et al. MicroRNA signatures differentiate preserved from reduced ejection fraction heart failure. Eur I Heart Fail 2015;17:405-15.

50 Sucharov CC, Kao DP, Port JD, et al. Myocardial microRNAs associated with reverse remodeling in human heart failure. JCI Insight 2017;2:e89169.

51 Hellawell JL, Margulies KB. Myocardial reverse remodeling. Cardiovasc Ther 2012:30:172-81.

52 Drakos SG, Pagani FD, Lundberg MS, et al. Advancing the science of myocardial recovery with mechanical circulatory support: a working group of the National, heart, lung and blood Institute. J Card Fail 2017;23:416-21.

53 Marfella R, Di Filippo C, Potenza N, et al. Circulating microRNA changes in heart failure patients treated with cardiac resynchronization therapy: responders vs. nonresponders. Eur J Heart Fail 2013:15:1277-88.

54 Melman YF, Shah R, Danielson K, et al. Circulating MicroRNA-30d is associated with response to cardiac resynchronization therapy in heart failure and regulates cardiomyocyte apoptosis: a translational pilot study. Circulation 2015;131:2202-16.

55 Bishu K, Deswal A, Chen HH, et al. Biomarkers in acutely decompensated heart failure with preserved or reduced ejection fraction. Am Heart J 2012;164:763-70.

56 Olivetti G, Quaini F, Sala R, et al. Acute myocardial infarction in humans is associated with activation of programmed myocyte cell death in the surviving portion of the heart. J Mol Cell Cardiol 1996;28:2005-16.

57 Vulesevic B, Lavoie SS, Neagoe P-E, et al. CRP induces NETosis in heart failure patients with or without diabetes. Immunohorizons 2019;3:378-88.

58 de Nigris F, Cacciatore F, Mancini FP, et al. Epigenetic hallmarks of fetal early atherosclerotic lesions in humans. JAMA Cardiol 2018;3:1184-91.

59 Grimaldi V, De Pascale MR, Zullo A, et al. Evidence of epigenetic tags in cardiac fibrosis. J Cardiol 2017;69:401-8.

60 Schiano C, Benincasa G, Franzese M, et al. Epigenetic-sensitive pathways in personalized therapy of major cardiovascular diseases. Pharmacol Ther 2020;210:107514

61 Napoli C, Benincasa G, Donatelli F, et al. Precision medicine in distinct heart failure phenotypes: focus on clinical epigenetics. Am Heart J 2020;224:113-28.

62 Benincasa G, Marfella R, Della Mura N, et al. Strengths and opportunities of network medicine in cardiovascular diseases. Circ J 2020;84:144-52. 